\title{
Automatic quenching of $\gamma$-ray emission in compact astrophysical sources
}

\author{
MARIA PETROPOULOU ${ }^{1}$ \\ APOSTOLOS MASTICHIADIS ${ }^{2}$ \\ Section of Astronomy, Astrophysics and Mechanics, Department of Physics, University of \\ Athens, Panepistimiopolis Zografos \\ Athens, Greece \\ 1 maroulaaki@gmail.com \\ 2 amastich@phys.uoa.gr \\ Received Day Month Year \\ Revised Day Month Year
}

\begin{abstract}
We investigate automatic $\gamma$-ray photon quenching in compact non-thermal sources. This is an auto-regulating network of processes that consists of photon-photon absorption and synchrotron emission of the produced $e^{-} e^{+}$pairs and operates non linearly whenever the $\gamma$-ray luminosity exceeds a critical value. We present expressions for this quantity and discuss our results.
\end{abstract}

Keywords: gamma-ray sources (astronomical); radiation mechanisms: non-thermal, synchrotron

PACS numbers: 98.70.Rz, 41.60.Ap

\section{Introduction}

In the present work we investigate under which conditions a compact magnetized source of $\gamma$-rays can sustain pair-production in an autonomous manner, extending previous calculations of Ref. 1 . If the luminosity of $\gamma$-rays exceeds a certain value, even in the case where no soft photons are initially present in the source, these appear automatically in the system. The non-linear network of processes that makes this possible is annihilation of $\gamma$-rays on synchrotron soft photons emitted by the created pairs.

\section{Physical processes and physical conditions}

We assume a spherical source of radius $R$ with embedded magnetic field $B$, where $\gamma$-rays are being injected uniformly throughout the source with luminosity $L_{\gamma}^{\text {inj. We }}$ define the photon $\gamma$-ray compactness as $l_{\gamma}^{\text {inj }}=\frac{L_{\gamma}^{\text {inj }} \sigma_{T}}{4 \pi R m_{e} c^{3}}$. The only physical processes taken into account are photon-photon absorption and synchrotron radiation - to facilitate our analytical calculations we have omitted inverse compton scattering; 
this however was icluded in our numerical treatment of the problem ${ }^{2}$. If the $\gamma$ ray compactness exceeds a certain critical value, then the following loop starts operating:

(i) $\gamma$-rays pair-produce on soft photons, which initially can be arbitrarily low inside the source.

(ii) The produced electron-positron pairs cool by emitting synchrotron radiation, thus acting as a source of soft photons.

(iii) The soft photons serve as targets for more $\gamma \gamma$-interactions.

The three-step process outlined above operates only in case where two conditions are simultaneously satisfied. The first one, which is a feedback criterion, requires that the synchrotron photons emitted from the pairs have enough energy to pair-produce on the $\gamma$-rays - we note that, throughout the text we use normalized photon and electron energies with respect to $m_{e} c^{2}$. If one combines, (i) the threshold condition for $\gamma \gamma$-absorption $\epsilon_{\gamma} \epsilon_{0} \geq 2$, (ii) the fact that there is equipartition of energy between the electron and the positron of the produced pairs, i.e. $\gamma_{e^{-}}=\gamma_{e^{+}}=\epsilon_{\gamma} / 2$, which is true at the threshold of pair production, and (iii) the assumption that the required soft photons of energy $\epsilon_{0}$ are the the synchrotron photons emitted by the pairs, i.e. $\epsilon_{0}=b \gamma_{e}^{2}$, where $b=B / B_{c r}$ and $B_{c r}=4.41 \times 10^{13} \mathrm{G}$, one derives the minimum value of the magnetic field required for quenching to become possible, i.e. $B_{q}=8 \epsilon_{\gamma}^{-3} B_{c r}$.

Quenching of $\gamma$-rays can enter the non linear regime and become fully operative, only if a second criterion is imposed, which is related to the optical depth for $\gamma \gamma$ absorption. A simple way to see this is with the following consideration. Assume that the $\gamma$-rays pair-produce on some soft photon and that each particle created cools by emitting several synchrotron photons. The critical condition occurs if the number density of the $\gamma$-rays (or equivalently $l_{\gamma}^{\text {inj }}$ ) is such that at least one of the emitted synchrotron photons, pair-produces on a $\gamma$-ray photon instead of escaping from the source. The second condition is the one that determines the critical $\gamma$-ray compactness $l_{\gamma, \mathrm{cr}}^{\mathrm{inj}}$.

\section{Critical $\gamma$-ray compactness - analytical results}

Below we give a general outline of the procedure for determining $l_{\gamma, \mathrm{cr}}^{\mathrm{inj}}$. For a more detailed description see Ref. 2.

(i) We begin by writing the kinetic equations that describe the distributions of $\gamma$-rays, soft photons and electrons in the source. In all our analytical calculations we have treated synchrotron emissivity as a $\delta$-function. Our work though expands previous calculations of Ref. 1 by treating synchrotron cooling as a differential loss operator.

(ii) By adding perturbations to the number densities of electrons and soft photons, we examine the stability of the trivial stationary solution, i.e. no soft photons and electrons are initially present in the source, whereas monoenergetic $\gamma$-rays are being constantly injected. 
(iii) We determine $l_{\gamma, \text { cr }}^{\text {inj }}$ by requiring equality of compactnesses of the perturbed soft and $\gamma$-ray photon distributions. Any further increase of the injected $\gamma$-ray luminosity will lead, on the one hand, to further increase of the soft photon luminosity and, on the other hand, to saturation of the $\gamma$-ray luminosity.

(iv) The calculations are performed while using two approximations for the cross section for $\gamma \gamma$-absorption. The results are shown below:

$$
\begin{aligned}
& l_{\gamma, \mathrm{cr}}^{\mathrm{inj}}=\sqrt{2 b \epsilon_{\gamma}} \quad \text { for } \quad \sigma_{\gamma \gamma}=\frac{\sigma_{T} x}{3} \delta\left(x-2 / \epsilon_{\gamma}\right) \\
& l_{\gamma, \mathrm{cr}}^{\mathrm{inj}}=\frac{3 b^{2} \epsilon_{\gamma}^{2}}{8}\left[\left(\frac{b \epsilon_{\gamma}}{2}\right)^{3 / 2}-\frac{2}{\epsilon_{\gamma}^{3}}\right]^{-1} \text { for } \quad \sigma_{\gamma \gamma}=\frac{4 \sigma_{T}}{3 x \epsilon_{\gamma}} \Theta\left(x \epsilon_{\gamma}-2\right)
\end{aligned}
$$

where $x$ is the dimensionless energy of the soft photons.

We have tested the validity of our analytical expressions (see Eqs.1 and 2) against those obtained with a numerical code. The result of the comparison in the case of the step-function approximation is shown in Fig. 1. If no approximations are made, the critical curve can be found only numerically. It is worth noting, that despite the simplifying assumptions used in our analytical treatment, the obtained function given by Eq.(2) succeeds in reproducing the behavior of the critical curve of the full problem. This allows us to use the analytical expression of Eq.(2) as a first order approximation to the one resulting from the full problem that can be obtained only after time-consuming numerical runs.

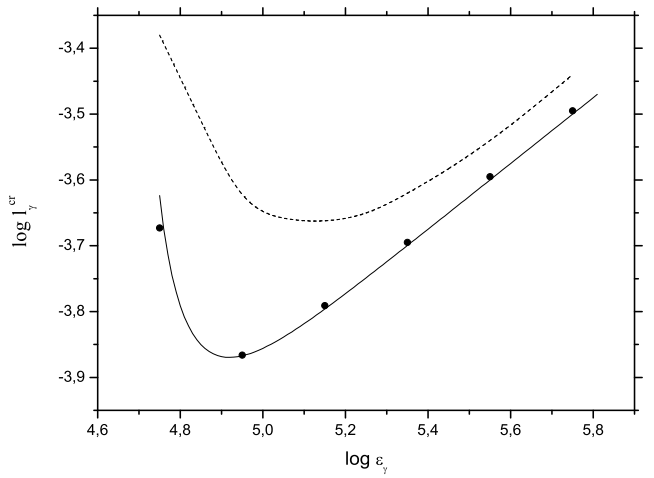

Fig. 1. Critical $\gamma$-ray compactness as a function of the injected $\gamma$-ray photon energy for $B=3.57$ G. The expression of Eq.(2) is plotted with solid line, while the points are the results of a numerical run using the same approximations. If no approximations are made on the synchrotrom emissivity and photon-photon cross section, the critical curve (dashed line) can be found only numerically.

\section{Critical $\gamma$-ray compactness in the case of a power-law injected spectrum}

As a next step, we study the effects that quenching has on a power-law spectrum of injected $\gamma$-rays (see Fig. 2). In all our numerical calculations the full expressions for $\sigma_{\gamma \gamma}$ and the synchrotron emissivity have been used. In the numerical treatment of the problem, synchrotron cooling is treated as a differential loss operator. 
If the injected $\gamma$-ray compactness is below the critical one, then the loop of processes described in Section 1 does not operate and the injected spectrum of $\gamma$-rays cannot be quenched (dashed lines in Fig. 2). For compactnesses above the critical one (solid lines in Fig. 2), quenching of $\gamma$-rays becomes dominant, redistributing energy from high to low energies. Thus, a break is produced in the injected powerlaw spectrum. If all photons with $\epsilon_{\gamma}>\epsilon_{\gamma}^{\min }$ satisfy the energy threshold criterion, quenching affects the whole $\gamma$-ray spectrum (panel (b) of Fig. 2). In any other case the high energy spectrum is partially affected (panel (a) of Fig. 2).

We also investigate the relation between the slope of the injected $\left(\alpha_{\text {inj }}\right)$ and the 'quenched' ( $\left.\alpha_{\text {que }}\right) \gamma$-ray spectrum, above the minimum absorption energy. For this reason, we ran the code for different $\alpha_{\text {inj }}$, choosing each time suitable values of the source parameters, to enable quenching. We find that $\alpha_{\text {que }} \propto \alpha_{\mathrm{inj}}^{1.34}$. Thus, the effects of quenching become more pronounced as the injected spectrum gets steeper. For steep enough injected spectra, e.g. with $\alpha_{\text {inj }}=2.8$, we can expect spectral breaks of the order $\Delta \alpha \approx 1.3$ in the high energy $\gamma$-ray spectrum.

(a)

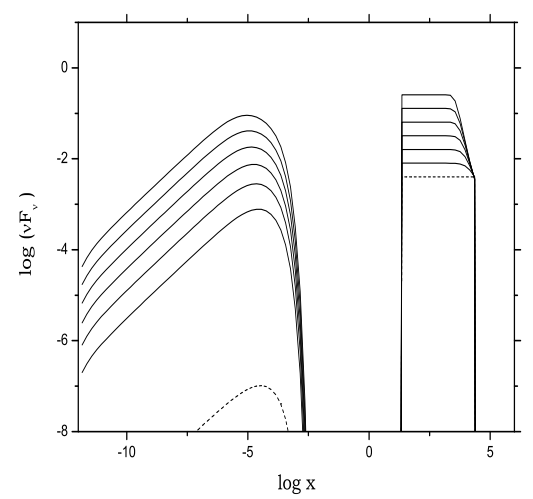

(b)

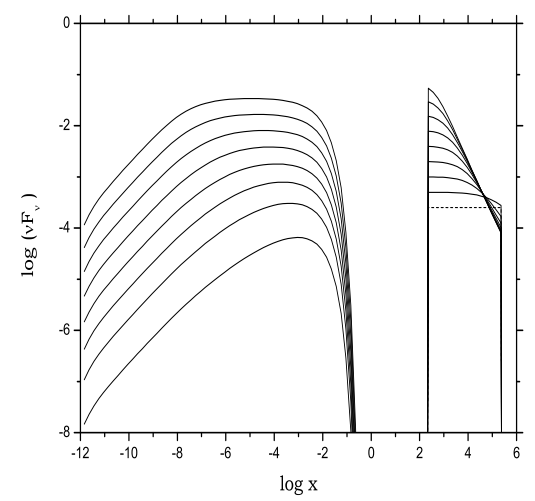

Fig. 2. MW spectra for a power-law injection of $\gamma$-rays of the form $Q_{\gamma}=Q_{0} \epsilon_{\gamma}^{-2}$ for $\epsilon_{\gamma}^{\min }<\epsilon_{\gamma}<$ $\epsilon_{\gamma}^{\max }$ and different values of the normalization $Q_{0}$. The parameters used in panels (a) and (b) respectively are: $\epsilon_{\gamma}^{\min }=23, \epsilon_{\gamma}^{\max }=2.3 \times 10^{4}, Q_{0}=4 \times 10^{-3}$ and $\epsilon_{\gamma}^{\min }=230, \epsilon_{\gamma}^{\max }=2.3 \times 10^{5}$, $Q_{0}=2.5 \times 10^{-4}$. In both cases, we start from $Q_{0}$, which then increases over each previous value by a factor of 2 . The dashed line curves represent the runs without quenching. For both cases, the source parameters are $R=3 \times 10^{16} \mathrm{~cm}$ and $B=40 \mathrm{G}$.

\section{Acknowledgments}

This research has been co-financed by the European Union (European Social Fund - ESF) and Greek national funds through the Operational Program 'Education and Lifelong Learning' of the National Strategic Reference Framework (NSRF) Research Funding Program: Heracleitus II. Investing in knowledge society through the European Social Fund. 


\section{References}

1. L. Stawarz and J. G. Kirk, ApJ 661, L17 (2007)

2. M. Petropoulou and A. Mastichiadis, $A \mathscr{E} A$ A 532, 11 (2011) 